\title{
Contraction Analysis of Nonlinear Distributed Systems
}

\author{
Winfried Lohmiller and Jean-Jacques E. Slotine \\ Nonlinear Systems Laboratory \\ Massachusetts Institute of Technology \\ Cambridge, Massachusetts, 02139, USA \\ wslohmil@mit.edu,jjs@mit.edu
}

\begin{abstract}
Contraction theory is a recently developed dynamic analysis and nonlinear control system design tool based on an exact differential analysis of convergence. This paper extends contraction theory to local and global stability analysis of important classes of nonlinear distributed dynamics, such as convection-diffusion-reaction processes, Lagrangian and Hamilton-Jacobi dynamics, and optimal controllers and observers.

The Hamilton-Jacobi-Bellman controller and a similar optimal nonlinear observer design are studied. Explicit stability conditions are given, which extend the well-known conditions on controllability and observability Grammians for linear time-varying systems.

Stability of the Hamilton-Jacobi dynamics is assessed by evaluating the Hessian of the system state along system trajectories. In contrast to stability proofs based on energy dissipation, this principle allows to conclude on stability of energy-based systems that are excited by time-varying inputs. In this context, contraction can be regarded as describing new variational conservation laws and the stability of entropy producing processes.
\end{abstract}




\section{Introduction}

This paper shows how the tools of contraction theory can contribute to the stability and convergence analysis of nonlinear partial differential processes [5, 7, 16, 15]. Contraction theory is a recently developed analysis and control system design tool based on an exact differential analysis of convergence. While differential approximation is the basis of all linearized stability analysis, what is new in contraction theory is that differential stability analysis can be made exact, and in turn yield global results on the nonlinear system [17, 18].

Specifically, the paper considers stability and convergence analysis of vector nonlinear diffusion processes and Hamiltonian dynamics, and more broadly of nonlinear partial differential equations in the general form

$$
\frac{\partial \phi_{i}}{\partial t}+h_{i}\left(\Phi, \nabla \phi_{i}, \vec{x}, t\right)=\nabla \cdot \mathbf{G}_{i}(\nabla \Phi, \vec{x}, t)
$$

where $\Phi$ is the $n$-dimensional continuous state vector (whose components $\phi_{i}$ consist, for instance, of chemical concentrations or mechanical velocities), and $\vec{x}$ is the $m$-dimensional vector of underlying coordinates. Specific application examples are then discussed, including distributed chemical processes, classical fluid systems such as Bernoulli and Navier-Stokes dynamics, Lagrangian systems, Hamilton-Jacobi dynamics, and Hamilton-Jacobi-Bellman designs of optimal controllers or observers [1, 4, 7]. We also show that approximations of such processes using basis functions are themselves contracting, regardless of the coarseness of the approximation.

Basically, a nonlinear dynamic system is called contracting if initial conditions or temporary disturbances are forgotten exponentially fast, i.e., if trajectories of the perturbed system return to their nominal behavior with an exponential convergence rate. In many physical systems this behavior can be interpreted as entropy producing - when the state corresponds to a physical quantity as velocity, temperature or a chemical concentration, the variation between actual and nominal behavior uses up available energy. As shown in [18] (to which the reader is referred for more details), it turns out that, for ordinary differential equations, relatively simple conditions can be given for this stability-like property to be verified, and furthermore that this property is preserved through basic system combinations, such as parallel combinations, series or hierarchies, and specific feedback combinations.

In order to extend these results to the distributed case (1), we will regard any distributed quantity in the following as a limit of a spatial discretization. Accordingly the $\nabla$-operator will be regarded as a limit of an explicit differentation and the $\int$-operator as a limit of a sum. Particular instances of these extensions were obtained in [19, 20], and in [13, 14] using operator notation, which we adopt here. In the following $\nabla$. corresponds to a inner product with the $\nabla$ operator, while $\nabla$ represents an outer product with the $\nabla$ operator.

Section 2 analyzes the contraction behavior of nonlinear first-order partial differential equations and their numerical solution, and discusses specific applications. Section 3 studies similarly second-order partial differential equations. Brief concluding remarks are offered in section 4 


\section{First-order partial differential equations}

This section concentrates on first-order nonlinear partial differential equations on a continuum $V$, such as e.g. Hamilton-Jacobi equations, in the general form

$$
\frac{\partial \phi_{i}}{\partial t}+h_{i}\left(\Phi, \nabla \phi_{i}, \vec{x}, t\right)=0
$$

Motivated by contraction analysis of ordinary systems in [18], consider two neighboring solutions of (2) at fixed time $t$, and the virtual displacement $\delta \Phi$. This leads to the virtual dynamics

$$
\frac{\partial}{\partial t} \delta \phi_{i}+\frac{\partial h_{i}}{\partial \Phi} \delta \Phi+\frac{\partial h_{i}}{\partial \nabla \phi_{i}} \delta \nabla \phi_{i}=0
$$

In most physical and technical applications, such as Bernoulli, Navier-Stokes and Hamilton-Jacobi systems, the term $\frac{\partial h_{i}}{\partial \nabla \phi_{i}}$ corresponds to an underlying velocity field of the quantity $\phi_{i}$ in (2), and so we will refer to this term as the flow "velocity".

Existence and uniqueness questions in nonlinear partial differential equations are notoriously difficult [7, 5, 16]. Because the solution of (1) or (2) needs not be differentiable with respect to $\vec{x}$, $\nabla \phi$ has to be carefully defined. We choose to define $\nabla \phi_{i}$ causally along flow lines, i.e., so that it corresponds, in the sense of distributions, to the limit of an explicit backward differentiation against the corresponding velocity component $\frac{\partial h_{i}}{\partial \nabla \phi_{i}}$ (in the case that a component is zero, we can choose the direction of the differentiation arbitrarily without affecting (3)). Accordingly we assume $\phi_{i}$ to be given at all inflowing boundaries, i.e., parts of the continuum boundary $\partial V$ where $\frac{\partial h_{i}}{\partial \nabla \phi_{i}} \mathbf{n}<0$, with $\mathbf{n}$ the unit vector along the outward normal. Interpreting (2) as an ordinary infinitesimal discretization in space can then lead to a natural existence condition on the solution of (1).

In this section, we derive two main results, one for a fixed continuum, and another for a continuum moving and deforming according to a predefined velocity field in $\vec{x}$ space. Applications of the first result includes transport laws and Bernoulli dynamics, while applications of the second includes Hamilton-Jacobi dynamics and optimal controller/observer design.

\subsection{First-order p.d.e.'s on a fixed continuum}

Let us analyze the contraction behavior of (3) on a fixed region $V$. We first derive the main result using partial integration, under the assumption that $\delta \phi_{i}$ and $\frac{\partial h_{i}}{\partial \nabla \phi_{i}}$ are continuously differentiable, before extending it to possibly discontinuous $\delta \phi_{i}$ and $\frac{\partial h_{i}}{\partial \nabla \phi_{i}}$ using a more technical proof.

We start with the flux equation, for a continuum $V$ of boundary $\partial V$,

$$
\int_{V} \nabla \cdot\left(\delta \phi_{i} \frac{\partial h_{i}}{\partial \nabla \phi_{i}} \delta \phi_{i}\right) d V=\int_{\partial V} \delta \phi_{i} \frac{\partial h_{i}}{\partial \nabla \phi_{i}} \mathbf{n} \delta \phi_{i} d \partial V
$$


where $\mathbf{n}$ is the unit vector along the outward normal of $\partial V$, and $\frac{\partial h_{i}}{\partial \nabla \phi_{i}} \mathbf{n}$ the corresponding outward flux. Assuming that $\phi_{i}$ is given at all inflowing boundaries, and using

$$
\nabla \cdot\left(\delta \phi_{i} \frac{\partial h_{i}}{\partial \nabla \phi_{i}} \delta \phi_{i}\right)=2 \delta \phi_{i} \frac{\partial h_{i}}{\partial \nabla \phi_{i}} \delta \nabla \phi_{i}+\delta \phi_{i} \nabla \cdot \frac{\partial h_{i}}{\partial \nabla \phi_{i}} \delta \phi_{i}
$$

the time derivative of $\int_{V} \delta \phi_{i} \delta \phi_{i} d V$ can be computed with partial integration as

$$
\begin{aligned}
\frac{1}{2} \frac{d}{d t} \int_{V} \delta \phi_{i} \delta \phi_{i} d V & =\int_{V} \delta \phi_{i} \frac{\partial}{\partial t} \delta \phi_{i} d V=\int_{V} \delta \phi_{i}\left(-\frac{\partial h_{i}}{\partial \Phi} \delta \Phi-\frac{\partial h_{i}}{\partial \nabla \phi_{i}} \delta \nabla \phi_{i}\right) d V \\
& =\int_{V}-\delta \phi_{i} \frac{\partial h_{i}}{\partial \Phi} \delta \Phi+\delta \phi_{i} \frac{\nabla}{2} \cdot \frac{\partial h_{i}}{\partial \nabla \phi_{i}} \delta \phi_{i} d V-\frac{1}{2} \int_{\partial V} \delta \phi_{i} \frac{\partial h_{i}}{\partial \nabla \phi_{i}} \mathbf{n} \delta \phi_{i} d \partial V
\end{aligned}
$$

This yields the bound and main result

$$
\frac{1}{2} \frac{d}{d t} \int_{V} \delta \Phi^{T} \delta \Phi d V \leq \int_{V} \delta \Phi^{T}\left(-\frac{\partial \mathbf{h}}{\partial \Phi}+\frac{\nabla}{2} \cdot \frac{\partial \mathbf{h}}{\partial \nabla \Phi}\right) \delta \Phi d V \leq \lambda_{V} \int_{V} \delta \Phi^{T} \delta \Phi d V
$$

where $\lambda_{V}$ denotes the largest eigenvalue of the symmetric part of $-\frac{\partial \mathbf{h}}{\partial \Phi}+\frac{\nabla}{2} \cdot \frac{\partial \mathbf{h}}{\partial \nabla \Phi}$.

Assume now that $\lambda_{V}$ is uniformly strictly negative (i.e., $\exists \beta>0, \forall \Phi, \forall t \geq 0, \lambda_{V} \leq-\beta<$ 0 ) then from (4) any $\int_{V} \delta \Phi^{T} \delta \Phi d V$ converges exponentially to zero. As in contraction analysis for ordinary differential equations, this immediately implies, by path integration, that any finite difference between two arbitrary solutions converges exponentially to zero. Thus, as in stable linear time-invariant systems, the initial conditions are exponentially "forgotten."

In the case that $\delta \phi_{i}$ or $\frac{\partial h_{i}}{\partial \nabla_{j} \phi_{i}}$ is not continuously differentiable, inequality (4) can still be obtained using a more technical proof, as discussed in the Appendix.

The argumentation can be extended to the following cases:

- Consider again the dynamics (2) with an upper bounded $-\frac{\partial \mathbf{h}}{\partial \Phi}$ and a given initial condition $\Phi_{o}$. This implies that $\delta \Phi^{T} \delta \Phi$ remains equal to zero $\forall t \geq 0$, which implies uniqueness of the solution $\forall t \geq 0$.

- Apply now to system (2) a set of linear constraints in $\Phi$ or $\nabla \Phi$. Such a case may describe, for instance, mechanical systems with kinematic constraints, incompressible fluid flows, and chemical systems in partial equilibrium. The constrained dynamic equations take the form

$$
\frac{\partial \phi_{i}}{\partial t}+h_{i}\left(\Phi, \nabla \phi_{i}, \vec{x}, t\right)+p_{i}=0
$$

where the constraint terms $\mathbf{p}(\Phi, \nabla \Phi, \vec{x}, t)$ are orthogonal to the constraint plane. Interpreting the above as an ordinary limit of a spatial discretization, we can conclude from [18] that contraction is preserved.

In summary, 
Theorem 1 Consider the dynamics

$$
\frac{\partial \phi_{i}}{\partial t}+h_{i}\left(\Phi, \nabla \phi_{i}, \vec{x}, t\right)+p_{i}=0
$$

with time $t, m$-dimensional coordinates $\vec{x}$, n-dimensional state vector $\Phi, p$ constraint terms $\mathbf{p}$ orthogonal to p linear algebraic constraints in $\Phi$ and $\nabla \Phi$, and $\nabla$ in line $i$ defined as the limit of a backward differentation against the velocity $\frac{\partial h_{i}}{\partial \nabla \phi_{i}}$.

For given $\phi_{i}$ over all inflowing boundaries $\partial V$ (i.e. $\frac{\partial h_{i}}{\partial \nabla \phi_{i}} \mathbf{n}<0$ ) and uniformly negative definite $-\frac{\partial \mathbf{h}}{\partial \Phi}+\frac{1}{2} \nabla \cdot \frac{\partial \mathbf{h}}{\partial \nabla \Phi}$ the system converges exponentially to a single solution, independent of initial conditions. The convergence rate is $\left|\lambda_{V}\right|$, where $\lambda_{V}$ is the largest eigenvalue of the symmetric part of $-\frac{\partial \mathbf{h}}{\partial \Phi}+\frac{1}{2} \nabla \cdot \frac{\partial \mathbf{h}}{\partial \nabla \Phi}$.

In the autonomous case $\left(h_{i}=h_{i}\left(\Phi, \nabla \phi_{i}, \vec{x}\right)\right.$, and constant boundary conditions and algebraic constraints) and under the same conditions, the system converges exponentially to a unique steadystate $\frac{\partial \Phi}{\partial t}=\mathbf{0}$.

We will call a system contracting if Theorem 1] applies. Similarly, we call the system semicontracting for $-\frac{\partial \mathbf{h}}{\partial \Phi}+\frac{1}{2} \nabla \cdot \frac{\partial \mathbf{h}}{\partial \nabla \Phi} \leq 0$ and indifferent for $-\frac{\partial \mathbf{h}}{\partial \Phi}+\frac{1}{2} \nabla \cdot \frac{\partial \mathbf{h}}{\partial \nabla \Phi}=0$.

More general local versions of the above theorem can also be derived as in [18]. Note that as the examples illustrate, contraction behavior can be interpreted physically as entropy producing, since $\delta \phi$ could have been used to spend available energy instead, if the state corresponds to a physical quantity such as velocity, temperature, or chemical concentration.

Example 2.1: Consider the conservation of a quantity $\phi$ in an $m$-dimensional continuum $V$

$$
\frac{\partial \phi}{\partial t}+\vec{v}(\vec{x}, t) \nabla \phi=0
$$

with $\phi$ given at all inflowing boundaries. Computing

$$
-\frac{\partial h}{\partial \phi}+\frac{1}{2} \nabla \cdot \frac{\partial h}{\partial \nabla \phi}=\frac{1}{2} \nabla \cdot \vec{v}
$$

we conclude with Theorem 1 on contraction behavior for a compressing flow field with $\nabla \cdot \vec{v} \leq 0$, with minimal convergence rate $\left|\frac{\nabla \cdot \vec{v}}{2}\right|$.

Consider another typical conservation of $\phi$ in an $m$-dimensional continuum $V$

$$
\frac{\partial \phi}{\partial t}+\nabla \cdot(\phi \vec{v}(\vec{x}, t))=0
$$

with $\phi$ given at all inflowing boundaries and equilibrium points. Similarly to the above we can conclude on contraction behavior for a expanding flow field with $\nabla \cdot \vec{v} \geq 0$ and minimal convergence rate $\left|\frac{\nabla \cdot \vec{v}}{2}\right|$.

Note that in the case that the velocity is itself the output of a contracting system, as e.g. will be the case later in example 3.3 then the overall system constitutes a hierarchy of contracting systems, and as such is contracting [18].

Observer designs for conservation dynamics will be illustrated in example 3.5 and example 3.6 
Example 2.2: Consider the $m$-dimensional Bernoulli dynamics

$$
\frac{\partial \phi}{\partial t}+\frac{1}{2} \nabla \phi^{T} H^{-1}(\vec{x}, t) \nabla \phi+U(\vec{x}, t)+p=0
$$

with kinetic energy $\frac{1}{2} \nabla \phi^{T} H^{-1}(\vec{x}, t) \nabla \phi$, potential energy $U(\vec{x}, t)$, and $\phi$ given at all inflowing boundary conditions of the continuum. The constraint term $p$ (energy due to pressure) stems from the incompressibility condition

$$
\nabla_{H} \cdot H^{-1} \nabla \phi=0
$$

where $\nabla_{H}$ denotes the covariant derivative with respect to the metric $H(\vec{x}, t)$. This dynamics governs many physical phenomena in fluids, structural mechanics and electromagnetism. Since

$$
-\frac{\partial h}{\partial \phi}+\frac{1}{2} \nabla_{H} \cdot \frac{\partial h}{\partial \nabla \phi}=0
$$

the Bernoulli dynamics is indifferent.

Example 2.3: Approximation of continuous distributed processes is routinely performed for numerical simulation, as well as in machine learning and vision. Let us now briefly discuss approximating the state of a contracting system (2) as $\hat{\Phi}(\vec{x}, t)=\mathbf{w}(\vec{x}, t) \mathbf{a}$, using $n$ basis functions $\mathbf{w}(\vec{x}, t)$ and their coefficient vector $\mathbf{a}$. The approximation leads to

$$
\frac{\partial \hat{\Phi}}{\partial t}+\mathbf{h}(\hat{\Phi}, \nabla \hat{\Phi}, \vec{x}, t)+\mathbf{p}=\mathbf{0}
$$

where the constraint terms $\mathbf{p}(\hat{\Phi}, \nabla \hat{\Phi}, \vec{x}, t)$ refers to the error introduced by the approximation. Multiplying (5) from the left with $\mathbf{w}^{T}$ and integrating results in an udpate law for $\mathbf{a}$,

$$
\int_{V} \mathbf{w}^{T} \mathbf{w} d V \dot{\mathbf{a}}+\int_{V} \mathbf{w}^{T}\left(\mathbf{h}(\mathbf{w a}, \nabla \mathbf{w} \mathbf{a}, \vec{x}, t)+\frac{\partial \mathbf{w}}{\partial t} \mathbf{a}\right) d V=\mathbf{0}
$$

where we used the orthogonality condition $\int_{V} \mathbf{w}^{T} \mathbf{p} d V=\mathbf{0}$. The constraint terms can be computed by solving (5) analytically with $\hat{\Phi}(\vec{x}, t)=\mathbf{w}(\vec{x}, t) \mathbf{a}$ and (6).

While the above is standard, the key remark is that, since a basis function approximation $\hat{\Phi}(\vec{x}, t)=$ $\mathbf{w}(\vec{x}, t) \mathbf{a}$ can be interpreted as a linear constraint, contraction is preserved by the approximation (using the basic result in [18] on adding linear constraints to contracting systems). Note that the approximation technique can be extended to more general partial differential equations, with the same argumentation on the contraction behavior.

Also note that we can smoothly extend the basis functions set with additional basis functions $w_{i}(\vec{x}, t)$ at any time during the simulation, by initializing the corresponding coefficient to $a_{i}=0$. Conversely, the deletion of a basis function $w_{i}(\vec{x}, t)$ from the set will lead to an additional disturbance $w_{i}(\vec{x}, t) a_{i}$ and can hence only be accepted for small $\left|a_{i}\right|$.

Finally, note that Theorem 1 can be extended to the following cases: 
- Consider a spatial discretization of $V$. Then Theorem 1 can still be applied with a corresponding discretized $\nabla$-operator since the method of proof in the Appendix is based on such a discretization.

- Assume that the $\nabla$-operator is generalized from a Jacobian to a covariant derivative with respect to a symmetric metric $\mathbf{M}(\vec{x}, t)$. Within a local normal coordinate system [1] defined by the metric, the definition of $\nabla$ in (2) is unchanged (vanishing Christoffel term), so that Theorem 1 can be applied unchanged.

\subsection{First-order p.d.e.'s along flow lines}

Whereas the convergence of $\phi$ is important for p.d.e. 's on a fixed ontinuum the convergence of $\vec{x}$ is important when we consider the dynamics along flow lines e.g. for mechanical systems or optimal control.

To assess the contraction behavior of $\vec{x}$ let us compute the evolution of the Hessian of $\phi$ along the flow lines. We will see later on in the optimal observer and controller dynamics and for physical Hamiltonian systems that the definiteness of $\nabla \nabla \phi$ will play an important role in the contraction behavior of the ordinary plant dynamics, using [18].

Let us compute the first and second gradient of (2)

$$
\begin{aligned}
\frac{\partial \nabla \phi}{\partial t} & =-\frac{\partial h}{\partial \vec{x}}-\frac{\partial h}{\partial \nabla \phi} \nabla \nabla \phi \\
\frac{\partial \nabla \nabla \phi}{\partial t} & =-\frac{\partial^{2} h}{\partial \vec{x}^{2}}-\frac{\partial^{2} h}{\partial \vec{x} \partial \nabla \phi} \nabla \nabla \phi-\nabla \nabla \phi^{T} \frac{\partial^{2} h}{\partial \nabla \phi \partial \vec{x}}-\nabla \nabla \phi^{T} \frac{\partial^{2} h}{\partial \nabla \phi^{2}} \nabla \nabla \phi-\frac{\partial h}{\partial \nabla \phi} \nabla \nabla \nabla \phi
\end{aligned}
$$

Following system trajectories

$$
\dot{\mathbf{x}}=\frac{\partial h}{\partial \nabla \phi}
$$

leads to

$$
\begin{aligned}
\frac{d \nabla \phi}{d t} & =\frac{\partial \nabla \phi}{\partial t}+\frac{\partial \nabla \phi}{\partial \vec{x}} \frac{d \vec{x}}{d t}=-\frac{\partial h}{\partial \vec{x}} \\
\frac{d \nabla \nabla \phi}{d t} & =\frac{\partial \nabla \nabla \phi}{\partial t}+\frac{\partial \nabla \nabla \phi}{\partial \vec{x}} \frac{d \vec{x}}{d t} \\
& =-\frac{\partial^{2} h}{\partial \vec{x}^{2}}-\frac{\partial^{2} h}{\partial \vec{x} \partial \nabla \phi} \nabla \nabla \phi-\nabla \nabla \phi^{T} \frac{\partial^{2} h}{\partial \nabla \phi \partial \vec{x}}-\nabla \nabla \phi^{T} \frac{\partial^{2} h}{\partial \nabla \phi^{2}} \nabla \nabla \phi
\end{aligned}
$$

where the above is known as the ordinary momentum dynamics of a Hamiltonian (see e.g. [7]). Equation (9) generalizes the Riccati equation (see e.g. [4]) to nonlinear Hamiltonian systems.

Let us perform a modal decomposition of $\nabla \nabla \phi=\mathbf{X}^{\mathbf{T}} \Lambda \mathbf{X}$ with real eigenvalue matrix $\Lambda$ and orthonormal eigenvector matrix X. Equation (9) then takes the form

$\frac{d \Lambda}{d t}=-\mathbf{X} \frac{\partial^{2} h}{\partial \vec{x}^{2}} \mathbf{X}^{T}-\mathbf{X}\left(\frac{\partial^{2} h}{\partial \vec{x} \partial \nabla \phi} \mathbf{X}^{T}+\dot{\mathbf{X}}^{T}\right) \Lambda-\Lambda\left(\mathbf{X} \frac{\partial^{2} h}{\partial \nabla \phi \partial \vec{x}}+\dot{\mathbf{X}}\right) \mathbf{X}^{T}-\Lambda \mathbf{X} \frac{\partial^{2} h}{\partial \nabla \phi^{2}} \mathbf{X}^{T} \Lambda$ 
where the diagonal corresponds to $n$ decoupled scalar Riccati equations of the eigenvalues $\lambda$ in $\Lambda$.

Uniform positive definiteness of $\lambda$ (i.e. $\nabla \nabla \phi$ or convexity of $\phi$ ) can be shown $\forall t \geq 0$ with given initial uniformly positive definite $\lambda_{o}$ or $\nabla \nabla \phi_{o}$ if $\lambda$ moves away from 0 in the neighborhood $\lambda=0$ and always stays upper bounded.

Using the modular Riccati equation the first condition (moves away from 0 ) is verified if the diagonal elements

$$
{\frac{d^{j} \Lambda}{d t^{j}}}_{\Lambda=0}=\operatorname{diag}\left(\mathbf{X} L^{j} \frac{\partial^{2} h}{\partial \vec{x}^{2}} \mathbf{X}^{T}\right)
$$

are larger or equal to 0 for $j=1$ and become uniformly positive in one of the higher derivatives $(j>1)$ if the above diagonal elements are not uniformly positive already.

This is equivalent to requiring that $L^{1} \frac{\partial^{2} h}{\partial \vec{x}^{2}}$ is positve semi-definite and the remaining nullspace becomes uniformly positive in one of the higher derivatives $(j>1)$ before it eventually becomes negative. The Lie-derivatives are here defined as

$$
\begin{aligned}
L^{o} \frac{\partial^{2} h}{\partial \vec{x}^{2}} & =-\frac{\partial^{2} h}{\partial \vec{x}^{2}} \\
L^{j+1} \frac{\partial^{2} h}{\partial \vec{x}^{2}} & =-\frac{d}{d t}\left(L^{j} \frac{\partial^{2} h}{\partial \vec{x}^{2}}\right)-\frac{\partial^{2} h}{\partial \vec{x} \partial \nabla \phi} L^{j} \frac{\partial^{2} h}{\partial \vec{x}^{2}}-L^{j} \frac{\partial^{2} h}{\partial \vec{x}^{2}} \frac{\partial^{2} h}{\partial \nabla \phi \partial \vec{x}}
\end{aligned}
$$

The inverse Riccati dynamics is given as

$$
\frac{d \nabla \nabla \phi^{-1}}{d t}=\nabla \nabla \phi^{-1} \frac{\partial^{2} h}{\partial \vec{x}^{2}} \nabla \nabla \phi^{-1}+\nabla \nabla \phi^{-1} \frac{\partial^{2} h}{\partial \vec{x} \partial \nabla \phi}+\frac{\partial^{2} h}{\partial \nabla \phi \partial \vec{x}} \nabla \nabla \phi^{-1}+\frac{\partial^{2} h}{\partial \nabla \phi^{2}}
$$

Performing similarly to the above a modular decomposition, the second condition (boundedness) is verified if the diagonal elements

$$
{\frac{d^{j} \Lambda^{-1}}{d t^{j}}}_{\Lambda=0}=\operatorname{diag}\left(\mathbf{X}^{T} L^{j} \frac{\partial^{2} h}{\partial \nabla \phi^{2}} \mathbf{X}\right)
$$

are larger or equal to 0 for $j=1$ and become uniformly positive in one of the higher derivatives $(j>1)$ if the above diagonal elements are not uniformly positive already.

This is equivalent to requiring that $L^{1} \frac{\partial^{2} h}{\partial \nabla \phi^{2}}$ is positive semi-definite and the remaining nullspace becomes uniformly positive in one of the higher derivatives $(j>1)$ before it eventually becomes negative. The Lie-derivatives are here defined as

$$
\begin{aligned}
L^{o} \frac{\partial^{2} h}{\partial \nabla \phi^{2}} & =\frac{\partial^{2} h}{\partial \nabla \phi^{2}} \\
L^{j+1} \frac{\partial^{2} h}{\partial \nabla \phi^{2}} & =\frac{d}{d t}\left(L^{j} \frac{\partial^{2} h}{\partial \nabla \phi^{2}}\right)+\frac{\partial h}{\partial \nabla \phi \partial \vec{x}} L^{j} \frac{\partial^{2} h}{\partial \nabla \phi^{2}}+L^{j} \frac{\partial^{2} h}{\partial \nabla \phi^{2}} \frac{\partial h}{\partial \vec{x} \partial \nabla \phi}
\end{aligned}
$$

The following theorem summarizes this conservation law of the curvature of $\phi$ along system trajectories. 
Theorem 2 Consider the dynamics

$$
\frac{\partial \phi}{\partial t}+h(\nabla \phi, \vec{x}, t)=0
$$

with time $t, m$-dimensional coordinates $\vec{x}$, and action $\phi$. Along trajectories, which move with flow velocity $\dot{\mathbf{x}}=\frac{\partial h}{\partial \nabla \phi}$, the Hessian $\nabla \nabla \phi$ evolves as

$$
\frac{d \nabla \nabla \phi}{d t}=-\frac{\partial^{2} h}{\partial \vec{x}^{2}}-\frac{\partial^{2} h}{\partial \vec{x} \partial \nabla \phi} \nabla \nabla \phi-\nabla \nabla \phi^{T} \frac{\partial^{2} h}{\partial \nabla \phi \partial \vec{x}}-\nabla \nabla \phi^{T} \frac{\partial^{2} h}{\partial \nabla \phi^{2}} \nabla \nabla \phi
$$

An initial convex $\phi$ (i.e. $\nabla \nabla \phi_{o}>0$ ) remains convex if conditions (10) and (12) are fulfilled.

Note that the conditions generalize the standard controllability Grammian condition [11] to general nonlinear Hamiltonian systems. Furthermore, they are explicitly computable, in contrast to the controllability Grammian which requires the unknown transition matrix.

Example 2.4: Consider the plant

$$
\frac{d \vec{x}}{d t}=\vec{f}(\vec{x}, \vec{u}, t)
$$

with $m$-dimensional state $\vec{x}, p$-dimensional control input $\vec{u}$, and cost-to-go dynamics

$$
\frac{d \phi}{d t}=-\ell(\vec{x}, \vec{u}, t) \leq 0
$$

along the plant trajectory with final cost $\phi_{f}(\vec{x})$ at time $t_{f}$. A typical example is $\ell=\frac{1}{2}\left(\vec{x}-\vec{x}_{d}\right)^{T} R(\vec{x}-$ $\left.\vec{x}_{d}\right)+\frac{1}{2}\left(\vec{u}-\vec{u}_{d}\right)^{T} Q\left(\vec{u}-\vec{u}_{d}\right)$, with desired trajectory $\vec{x}_{d}(t)$, supporting open-loop input $\vec{u}_{d}$, and constant uniformly positive definite $R$ and $Q . \phi$ can be minimized locally by minimizing the Hamiltonian

$$
h=\ell(\vec{x}, \vec{u}, t)+\nabla \phi \vec{f}(\vec{x}, \vec{u}, t)
$$

over $\vec{u}$ leading to $\frac{\partial \ell}{\partial \vec{u}}=\nabla \phi \frac{\partial \vec{f}}{\partial \vec{u}}$. This leads to the well known Hamilton-Jacobi-Bellman equation [4]

$$
\begin{aligned}
\frac{\partial \phi}{\partial t}+h(\nabla \phi, \vec{x}, t) & =0 \\
\frac{d \vec{x}}{d t} & =\frac{\partial h}{\partial \nabla \phi}
\end{aligned}
$$

on a continuum $V$ which moves with $\frac{d \vec{x}}{d t}$. This equation is solved backwards in time from final time $t_{f}$. Assuming $\phi_{f}\left(t_{f}\right)$ to be convex Theorem 2 allows to compute under which condition $\phi$ stays convex $\forall t<t_{f}$. As a result Theorem 2 allows to conclude when a global minium of $\phi$ is given by (13).

The ordinary contraction behavior of this optimal control design can be computed with the variation of (7)

$$
\delta \dot{\vec{x}}=\left(\frac{\partial^{2} h}{\partial \nabla \phi^{2}} \nabla \nabla \phi+\frac{\partial^{2} h}{\partial \nabla \phi \partial \vec{x}}\right) \delta \vec{x}
$$


and using (9) and (12)

$$
\frac{d}{d t}\left(\delta \vec{x}^{T} \nabla \nabla \phi \vec{x}\right)=-\delta \vec{x}^{T}\left(\frac{\partial^{2} h}{\partial \vec{x}}-\nabla \nabla \phi \frac{\partial^{2} h}{\partial \nabla \phi^{2}} \nabla \nabla \phi\right) \delta \vec{x} \leq \delta \vec{x}^{T} L^{1} \frac{\partial^{2} h}{\partial \vec{x}} \delta \vec{x}
$$

We can conclude on (semi)-contraction behavior (i.e. uniformly positive definite $\nabla \nabla \phi$ and negative (semi)-definite $L^{1} \frac{\partial^{2} h}{\partial \vec{x}}$ ) if the conditions in (10) and (12) are fulfilled for $j=1(j \geq 1)$. Note that asymptotic convergence can even be guaranteed for semi-definite $L^{1} \frac{\partial^{2} h}{\partial \vec{x}}$ since the differential length cannot get stuck due to the higher Lie derivatives in 10 .

In the case that the Hamiltonian dynamics is solved numerically, e.g. with basis functions or a neuronal network the above argumentation stays unchanged if the Hamiltonian is extended with the constraint term in (5).

If $u$ cannot be computed analytically, but rather numerically through a convex minimization process, then the overall dynamics is still indifferent, since it then corresponds to a hierarchy [18] composed of a contracting convex minimization process and an indifferent Hamiltonian dynamics.

Example 2.5: Consider again the plant

$$
\frac{d \vec{x}}{d t}=\vec{f}(\vec{x}, \vec{u}, t)
$$

with $m$-dimensional state $\vec{x}, p$-dimensional measurement feedback $\vec{u}$, and cost dynamics

$$
\frac{d \phi}{d t}=\ell(\vec{x}, \vec{u}, t) \geq 0
$$

with initial cost $\phi_{o}(\vec{x})$ at time $t_{o}$. A typical example is $\ell=\frac{1}{2}\left(\vec{y}-\vec{y}_{m}\right)^{T} R\left(\vec{y}-\vec{y}_{m}\right)+\frac{1}{2} \vec{u}^{T} Q \vec{u}$, with measurement $\vec{y}_{m}$, measurement estimate $\vec{y}(\vec{x}, t)$, and constant uniformly positive definite $R$ and $Q$. $\phi$ can be minimized by minimizing a slightly different Hamiltonian

$$
h=-\ell(\vec{x}, \vec{u}, t)+\nabla \phi \vec{f}(\vec{x}, \vec{u}, t)
$$

over $\vec{u}$, leading to $\frac{\partial \ell}{\partial \vec{u}}=\nabla \phi \frac{\partial \vec{f}}{\partial \vec{u}}$. This leads again to the Hamilton-Jacobi equation

$$
\frac{\partial \phi}{\partial t}+h(\nabla \phi, \vec{x}, t)=0
$$

which has to be integrated now with $t$. This is a generalization of the well-known Hamilton-Jacobi controller design to nonlinear optimal observer design, with the optimal solution obtained at the minimum, where $\nabla \phi=\overrightarrow{0}$.

The convexity of $\phi$ and hence the global uniqueness of the mimimum of $\phi$ can be shown with Theorem 2. This result also hold for an approximated system if the constraint term is added to the Hamiltonian.

Les us now show the relation of this observer design to the well-known Kalman observer (see e.g. [11]) for linear time-varying systems. The optimal state estimate $\hat{x}$ can the be found at the minimum of $\phi$, i.e. for $\nabla \phi=\overrightarrow{0}$, leading with (14) to

$$
\frac{d \nabla \phi}{d t}=\frac{\partial \nabla \phi}{\partial t}+\frac{\partial \nabla \phi}{\partial \vec{x}} \frac{d \hat{x}}{d t}=-\frac{\partial h}{\partial \hat{x}}-\nabla \nabla \phi \frac{\partial h}{\partial \nabla \phi}+\nabla \nabla \phi \frac{d \hat{x}}{d t}=\overrightarrow{0}
$$


This yields the following observer design in $\hat{x}$

$$
\frac{d \hat{x}}{d t}=\frac{\partial h}{\partial \nabla \phi}+(\nabla \nabla \phi)^{-1} \frac{\partial h}{\partial \vec{x}}
$$

with $\nabla \phi=0$ and the inverse covariance matrix (or information matrix) $\nabla \nabla \phi$ defined in Theorem 2

\section{Second-order partial differential equations}

This section discusses second-order nonlinear partial differential equations, such as e.g. diffusion equations on a closed continuum $V$, of the form

$$
\frac{\partial \Phi}{\partial t}=\nabla \cdot \mathbf{G}(\nabla \Phi, \vec{x}, t)
$$

where $\Phi$ is the $n$-dimensional continuous state vector, $\vec{x}$ is the $m$-dimensional vector of underlying coordinates, and $t$ is time.

In most physical and technical applications such as diffusion and Lagrangian dynamics, the first $\nabla$ operator and the $\nabla$ operator in the argument correspond to a limit of a left and right differentation or vice versa. Accordingly either $\Phi$ or the projection of $\nabla \Phi$ needs to be given on $\partial V$, so that both $\nabla$ operators can be defined there.

Interpreting (15) under the above conditions as an ordinary infinitesimal discretization in space leads to a natural existence conditions for the solution of (1).

Similar to section 2 consider now two neighboring solutions of (2) at fixed time $t$, and the virtual displacement $\delta \Phi$ between them, leading to the virtual dynamics

$$
\frac{\partial}{\partial t} \delta \Phi=\nabla \cdot \frac{\partial \mathbf{G}}{\partial \nabla \Phi} \delta \nabla \Phi
$$

The time-derivative of $\int_{V} \delta \Phi^{T} \delta \Phi d V$ can be computed as

$$
\frac{1}{2} \frac{d}{d t} \int_{V} \delta \Phi^{T} \delta \Phi d V=-\int_{V} \delta \nabla \Phi^{T} \frac{\partial \mathbf{G}}{\partial \nabla \Phi} \delta \nabla \Phi d V
$$

where we have exploited that the transpose of a left-differentation corresponds to a negative right differentation. Lower bounding $\frac{\partial G_{i j}}{\partial \nabla_{k} \phi_{l}} \geq \Lambda_{i j k l} \geq 0$ with $\Lambda_{i j k l}=0$ for $i j \neq k l$ allows to conclude on semi-contraction behavior independent of the boundary conditions.

For given $\Phi$ on $\partial V$ the minimal contraction rate can be computed with a Fourier expansion as

$$
\frac{1}{2} \frac{d}{d t} \int_{V} \delta \Phi^{T} \delta \Phi d V \leq-\sum_{i=1}^{n} \sum_{j=1}^{m} \frac{\Lambda_{i j i j} \pi^{2}}{l_{i}^{2}} \int_{V} \delta \Phi^{T} \delta \Phi d V
$$


where $l_{i}$ is the maximum length of the continuum along the $i^{\text {th }}$ axis. By path integration, this immediately implies that any finite difference between two arbitrary solutions has an equivalent contraction behavior. Combining this result with Theorem 1] using the superposition principle [18] leads to

Theorem 3 Consider the dynamics

$$
\frac{\partial \phi_{i}}{\partial t}+h_{i}\left(\Phi, \nabla \phi_{i}, \vec{x}, t\right)+p_{i}=\nabla \cdot \mathbf{G}_{i}(\nabla \Phi, \vec{x}, t)
$$

with time $t, m$-dimensional coordinates $\vec{x}$, n-dimensional state vector $\Phi, p$ constraint terms $\mathbf{p}$ orthogonal to p linear algebraic constraints in $\Phi$ and $\nabla \Phi$, and $\nabla$ in line $i$ defined as the limit of a backward differentation against the velocity $\frac{\partial h_{i}}{\partial \nabla \phi_{i}}$.

Let $l_{i}$ be the diameter (maximum length) of the continuum along the $i^{\text {th }}$ axis and lower bound the positive semi-definite $\frac{\partial G_{i j}}{\partial \nabla_{k} \phi_{l}} \geq \Lambda_{i j k l} \geq 0$ for a (perhaps time-varying) Dirichlet condition (i.e., $\Phi(t)$ specified on $\partial V$ ) and define $\Lambda_{i j k l}=0$ for the remaining (perhaps time-varying) Neumann condition (i.e., $\nabla \Phi(t)$ specified along the normal of $\partial V$ ).

For given $\phi_{i}$ over all inflowing boundaries $\partial V$ (i.e. $\frac{\partial h_{i}}{\partial \nabla \phi_{i}} \mathbf{n}<0$ ) and uniformly negative definite $-\frac{\partial \mathbf{h}}{\partial \Phi}+\frac{1}{2} \nabla \cdot \frac{\partial \mathbf{h}}{\partial \nabla \Phi}$ the solution converges unique and exponentially to a single solution, independent of the initial conditions. The convergence rate is $\left|\lambda_{V}-\sum_{i=1}^{n} \sum_{j=1}^{m} \frac{\Lambda_{i j i j} \pi^{2}}{l_{i}^{2}}\right|$, where $\lambda_{V}$ is the largest eigenvalue of the symmetric part of $-\frac{\partial \mathbf{h}}{\partial \Phi}+\frac{1}{2} \nabla \cdot \frac{\partial \mathbf{h}}{\partial \nabla \Phi}$.

In the autonomous case ( $\mathbf{h}=\mathbf{h}(\Phi, \nabla \Phi, \vec{x}), \mathbf{G}=\mathbf{G}(\nabla \Phi, \vec{x})$ and with constant boundary conditions and algebraic constraints) and under the same conditions, the system converges exponentially to a unique steady-state $\frac{\partial \Phi}{\partial t}=0$.

We will call the system contracting if at least one of the two above conditions is fulfilled. We will call such systems semi-contracting if the above matrices are only semi-definite. And finally we will call them indifferent if the above matrices are skew-symmetric.

The method of proof implies that all the results on contracting systems in [18] and extensions from section 2 can be extended to contracting reaction-convection-diffusion processes, with boundary conditions acting as additional inputs to the system. For instance, any autonomous contracting reaction-convection-diffusion process, when subjected to boundary conditions periodic in time, will tend exponentially to a periodic solution of the same period. The convergence is robust to bounded or linearly increasing disturbances. Finally, the method of proof also implies that any regular spatial discretization of the above process is contracting as well, when the explicit differentaion is performed against the velocity.

Again, the following examples will illustrate that contraction behavior can be intpreted physically as entropy producing, since $\delta \phi$ could have been used to spend available energy instead.

Example 3.1: Consider a wafer disk, whose temperature is controlled with a continuous external light 
source [19]. The dynamic equations are

$$
\frac{\partial \phi}{\partial t}+h\left(\phi^{4}-\phi_{o}^{4}\right)=\nabla \cdot g^{*}(r) \nabla \phi \quad \text { with } r=\|\nabla \phi\| .
$$

with $\phi>0$ the wafer temperature, $\phi_{o}(t)$ the external temperature, $h$ a radiation constant, and given $\phi$ at the boundary of the disk. Assume that $g^{*} \geq 0$ and $\frac{\partial\left(g^{*} r\right)}{\partial r} \geq 0$ then the Jacobian

$$
\frac{\partial \mathbf{G}}{\partial \nabla \phi}=g^{*} \vec{I}+r \frac{\partial g^{*}}{\partial r} \frac{\nabla \phi}{\|\nabla \phi\|} \frac{\nabla \phi}{\|\nabla \phi\|}^{T} \geq{\frac{\partial\left(g^{*} r\right)}{\partial r}}_{\| \nabla \phi}^{\|\nabla \phi\|} \frac{\nabla \phi}{\|\nabla \phi\|}^{T}
$$

is positive semi-definite. A specific saturated diffusion term may be of the form $g^{*}=\tanh (\alpha r) /(\alpha r)$ (with $\alpha$ a constant), for instance.

Computing $-\frac{\partial h}{\partial \phi}+\frac{1}{2} \nabla \cdot \frac{\partial h}{\partial \nabla \phi}=-4 h \phi^{3}$, we conclude with Theorem 3 on contraction behavior with minimal convergence rate $4 h \phi^{3}$.

This result may be used to design observers for the system. Indeed, it means that an open-loop (identity) observer for the system will converges to the actual temperature distribution with minimal convergence rate $4 h \hat{\phi}^{3}$. Similarly, assume now that the actual temperature is measured at the boundary of the disk. Using this measurement as a boundary condition for the observer state increases the convergence rate according to Theorem 3 while at the same time preserving consistency with the actual plant (i.e., keeping the actual temperature as a particular solution of the observer equations with their boundary condition). Such designs are illustrated in [19] for a linear $g$.

Example 3.2: Consider the viscosity solution [7] of the Hamilton-Jacobi equation

$$
\frac{\partial \phi}{\partial t}+h(\nabla \phi, \vec{x}, t)=g \nabla^{2} \phi
$$

along a continuum $V$, which moves with the velocity $\frac{\partial h}{\partial \nabla \phi}$ and given $\phi$ or $\nabla \phi$ along the normal of $\partial V$. Computing

$$
-\frac{\partial h}{\partial \phi}+\frac{1}{2} \nabla \cdot \frac{\partial h}{\partial \nabla \phi}=0
$$

we can conclude with Theorem 3 on semi-contraction for $g \leq 0$. The minimal contraction rate of the dynamics is governed solely by the viscosity term, the geometry and the boundary conditions of the continuum $V$.

Example 3.3: Consider the $m$-dimensional Navier-Stokes equation

$$
\frac{\partial \mathbf{v}}{\partial t}+\nabla \mathbf{v} \mathbf{v}+\nabla U(\vec{x}, t)+\nabla p=g \nabla \cdot \nabla \mathbf{v}
$$

with velocity $\mathbf{v}$, potential energy $U(\vec{x}, t)$, viscosity $g$, incompressiblity condition $\nabla \cdot \mathbf{v}=0$ leading to a pressure gradient $\nabla p$ and $\mathbf{v}$ given at all boundaries. Computing

$$
-\frac{\partial \mathbf{h}}{\partial \mathbf{v}}+\frac{1}{2} \nabla \cdot \frac{\partial \mathbf{h}}{\partial \nabla \mathbf{v}}=\frac{1}{2} \nabla \mathbf{v}
$$


we can conclude with Theorem 3 on contraction behavior for positive definite $\nabla \mathbf{v}+\sum_{i=1}^{m} \frac{g \pi^{2}}{l_{i}^{2}} \mathbf{I}$, where $l_{i}$ is the diameter of the continuum in direction $i$. Accordingly, uniqueness of Navier-Stokes solutions can be concluded for bounded $\nabla \mathbf{v}$.

Comparing this result with Euler dynamics (i.e. $g=0$ ) we see that the stability is increased by the viscosity term. Comparing this result with the velocity potential $\phi$ in the Bernoulli dynamics in example 2.2 . potential instabilities of the Navier-Stokes can only be explained with vortices within the dynamics. This nonlinear result on a finite convergence region generalizes the well-known stability results on continuously differentiable infinitesimal small disturbances in [6].

Example 3.4: Consider a continuum $V$ with $\Phi$ or $\nabla \Phi$ given along the normal of $\partial V$, and the Lagrangian

$$
\mathscr{L}=T(\nabla \Phi, \vec{x}, t)-U(\Phi, \vec{x}, t)
$$

Assume that $\mathscr{L}$ is convex, i.e. that $\frac{\partial^{2} T}{\partial \Phi^{2}}$ and $-\frac{\partial^{2} U}{\partial \nabla \Phi^{2}}$ are both uniformly positive definite. Then $\mathscr{L}$ can be minimized using the Euler-Lagrange dynamics (see e.g. [4])

$$
\frac{\partial \Phi}{\partial t}+\frac{\partial \mathscr{L}}{\partial \Phi}=\nabla \cdot \frac{\partial \mathscr{L}}{\partial \nabla \Phi}
$$

Computing

$$
-\frac{\partial h}{\partial \Phi}+\frac{1}{2} \nabla \cdot \frac{\partial h}{\partial \nabla \Phi}=\frac{\partial^{2} U}{\partial \Phi^{2}}
$$

contraction can be concluded using Theorem 3

This result is especially useful in function approximation and image processing, where $-U$ describes e.g. the quadratic deviation between measured and estimated $\Phi$, and $T$ describes a nonlinear smoothing cost, e.g. penalizing small and medium gradients but letting through large gradients such as edges.

Example 3.5: In machine vision, consider an image (grey level) $\phi$, with $\phi$ given at all inflowing boundaries of the imaging plane, and $\nabla \phi$ given along the normal of the remaining boundaries. Consider the following combination of optical flow (conservation of brightness) from example 2.1] and Lagrangian dynamics from example 3.4 (see [22, 23]).

$$
\frac{\partial \phi}{\partial t}+\vec{v} \nabla \phi+\frac{\partial \mathscr{L}}{\partial \phi}=\nabla \cdot \frac{\partial \mathscr{L}}{\partial \nabla \phi}
$$

with given camera motion flow $\vec{v}(\vec{x}, t)$. Computing

$$
-\frac{\partial h}{\partial \phi}+\frac{1}{2} \nabla \cdot \frac{\partial h}{\partial \nabla \phi}=-\frac{\partial^{2} \mathscr{L}}{\partial \phi^{2}}+\frac{\nabla \cdot \vec{v}}{2}
$$

we can conclude with Theorem $\left[3\right.$ on contraction behavior for positive definite $\frac{\partial^{2} \mathscr{L}}{\partial \nabla \phi^{2}}$ and strictly negative $-\frac{\partial^{2} \mathscr{L}}{\partial \phi^{2}}+\frac{\nabla \cdot \vec{v}}{2}$. 
The approach can be vastly extended by allowing for a prior differential coordinate transformation in $\Phi$, as in [18]. Specifically, the line vector $\delta \Phi$ between two neighboring trajectories can also be expressed using the differential coordinate transformation $\delta \Psi=\Theta \delta \Phi$ where $\boldsymbol{\Theta}(\boldsymbol{\Phi}, \nabla \Phi, \vec{x}, t)$ is a square matrix. This leads to a generalization of our earlier definition of squared length

$$
\int_{V} \delta \boldsymbol{\Psi}^{T} \delta \boldsymbol{\Psi} d V=\int_{V} \delta \boldsymbol{\Phi}^{T} \mathbf{M} \delta \boldsymbol{\Phi} d V
$$

with metric $\mathbf{M}=\Theta^{T} \boldsymbol{\Theta}$. Note that in general we cannot expect to find explicit new coordinates $\boldsymbol{\Psi}(\boldsymbol{\Phi}, t)$, but $\delta \boldsymbol{\Psi}$ and $\delta \boldsymbol{\Psi}^{T} \delta \boldsymbol{\Psi}$ can always be defined. We require $\mathbf{M}$ to be uniformly positive definite, so that exponential convergence of $\delta \boldsymbol{\Psi}$ to $\mathbf{0}$ also implies exponential convergence of $\delta \boldsymbol{\Phi}$ to 0 . The following example illustrates the application of Theorem 3 to new coordinates $\delta \Psi$.

Example 3.6: Theorem 3 can be applied to a distributed version of an example studied in detail in [19]. Specifically, consider the temperature-dependent reaction $A \rightarrow B$ in an open reaction volume $V$ of size $100 \times 100$ units

$$
\frac{\partial}{\partial t}\left(\begin{array}{c}
c_{A} \\
T
\end{array}\right)+\vec{v}^{T}\left(\begin{array}{c}
\nabla c_{A} \\
\nabla T
\end{array}\right)=g \nabla \cdot\left(\begin{array}{c}
\nabla c_{A} \\
\nabla T
\end{array}\right)+\left(\begin{array}{c}
-1 \\
-100
\end{array}\right) e^{-\frac{E}{T}} c_{A}
$$

with $0 \leq c_{A} \leq 1$ the partial concentration of $A, T>0$ the measured temperature, $g>0$ the diffusion constant, $E>0$ the specific activation energy, and $\vec{v}(\vec{x}, t)$ the velocity field within the reaction chamber.

The reaction volume is open at the left and the right and closed at the top and the bottom. Accordingly we assume that $\nabla c_{A}=0$ and $\nabla T=0$ everywhere along the normal of the boundary, except for $x=0$ where we have $c_{A}=0$ and $T=500$. For $40 \leq y \leq 60$ the chemical $A$ is injected with concentration $c_{A f}(t)$ and temperature $T_{f}(t)$. Extending[19], the observer can be defined similarly as

$$
\frac{\partial}{\partial t}\left(\begin{array}{c}
\hat{c}_{A} \\
\hat{T}
\end{array}\right)+\vec{v}^{T}\left(\begin{array}{c}
\nabla \hat{c}_{A} \\
\nabla \hat{T}
\end{array}\right)=g \nabla \cdot\left(\begin{array}{c}
\nabla \hat{c}_{A} \\
\nabla \hat{T}
\end{array}\right)+\left(\begin{array}{c}
-1 \\
-100
\end{array}\right) e^{-\frac{E}{\hat{T}} \hat{c}_{A}}+\left(\begin{array}{c}
k_{1} \\
k_{2}
\end{array}\right) \int_{T}^{\hat{T}} e^{-\frac{E}{T}} d T
$$

An appropriate linear coordinate transformation with constant uniformly positive metric is computed in [19] for the ordinary reaction terms, based on a linear matrix inequality. Since the convection and diffusion terms do not change under such a linear coordinate transformation, Theorem 3 implies contraction behavior for both observer designs and the plant for $g \geq 0, \nabla \cdot \vec{v}=0$, and uniformly negative definite projection of the Jacobian $\frac{\partial \mathbf{h}}{\partial \Phi}$ on the new coordinates.

The corresponding system responses can be accessed in [25].

\section{Concluding Remarks}

This paper focused on the contraction analysis of important physical and engineered first and second-order distributed systems, such as Navier-Stokes, Euler, Lagrangian and Hamilton-Jacobi dynamics. In principle the technique can be extended to higher-order systems by repeating the partial integration. 


\section{Appendix: Derivation of inequality (4)}

If either $\delta \phi_{i}$ or $\frac{\partial h_{i}}{\partial \nabla_{j} \phi_{i}}$ is not continuously differentiable, then standard partial integration cannot be used in the derivation of inequality (4). The inequality can still be obtained using a more technical proof. Specifically, we compute the convection term $\frac{\partial h_{i}}{\partial \nabla_{j} \phi_{i}} \nabla_{j}$ in

$$
\frac{\partial}{\partial t} \delta \phi_{i}+\frac{\partial h_{i}}{\partial \Phi} \delta \Phi+\sum_{j} \frac{\partial h_{i}}{\partial \nabla_{j} \phi_{i}} \nabla_{j} \delta \phi_{i}=0
$$

based on the limit of a spatial discretization, where $\nabla$ is defined against the velocity component $\frac{\partial h_{i}}{\partial \nabla_{j} \phi_{i}}$, and lower bound this discretization matrix with the diagonal matrix $\frac{\nabla_{j}}{2} \cdot \frac{\partial h_{i}}{\partial \nabla_{j} \phi_{i}}$, so that we get inequality (4) again. This lower bound is obtained using the following computation

$$
\begin{aligned}
& \left(\frac{\partial h_{i}}{\partial \nabla_{j} \phi_{i}} \nabla_{j}-\frac{\nabla_{j}}{2} \cdot \frac{\partial h_{i}}{\partial \nabla_{j} \phi_{i}}\right) d x_{j}=\sum_{k}\left(-\left|\frac{\partial h_{i}}{\partial \nabla_{j} \phi_{i}}\right|+\left|\frac{\partial h_{i}}{\partial \nabla_{j} \phi_{i}}\right|-\left|\frac{\partial h_{i}}{\partial \nabla_{j} \phi_{i} k}\right|-\right)_{k, k-1 . . k+1} \\
& -\sum_{k} \frac{1}{2}\left(\begin{array}{ccc}
-\left|\frac{\partial h_{i}}{\partial \nabla_{j} \phi_{i}}\right|+ & 0 & 0 \\
0 & \left|\frac{\partial h_{i}}{\partial \nabla_{j} \phi_{i}}\right| & 0 \\
0 & 0 & -\left|\frac{\partial h_{i}}{\partial \nabla_{j} \phi_{i} k}\right|-
\end{array}\right)_{k-1 . . k+1, k-1 \ldots k+1} \\
& =\sum_{k}\left(\begin{array}{ccc}
\frac{1}{2}\left|\frac{\partial h_{i}}{\partial \nabla_{j} \phi_{i}}\right|+ & 0 & 0 \\
-\left|\frac{\partial h_{i}}{\partial \nabla_{j} \phi_{i}}\right|+ & \frac{1}{2}\left|\frac{\partial h_{i}}{\partial \nabla_{j} \phi_{i}}\right| & -\left|\frac{\partial h_{i}}{\partial \nabla_{j} \phi_{i}}\right|_{k} \\
0 & 0 & \frac{1}{2}\left|\frac{\partial h_{i}}{\partial \nabla_{j} \phi_{i}}\right| \mid-
\end{array}\right)_{k-1 . . k+1, k-1 . . k+1} \\
& \geq 0
\end{aligned}
$$

with discretization index $k,\left|\frac{\partial h_{i}}{\partial \nabla_{j} \phi_{i}}\right|_{+}=\max \left(\frac{\partial \mathrm{h}_{\mathrm{i}}}{\partial \nabla_{\mathrm{j}} \phi_{\mathrm{i}}}, 0\right),\left|\frac{\partial h_{i}}{\partial \nabla_{j} \phi_{i}}\right|_{-}=\max \left(-\frac{\partial \mathrm{h}_{\mathrm{i}}}{\partial \nabla_{\mathrm{j}} \phi_{\mathrm{i}}}, 0\right)$, and where only the non-zero elements of the discretization matrices are shown. In the case that $\phi_{i}$ is given either at $k+1$ or $k-1$, then the corresponding row and column must be deleted, without any impact on the definiteness.

\section{References}

[1] Aris, R., Vectors, Tensors, and the Basic Equations of Fluid Mechanics, Dover Publications, (1962).

[2] Arnold, V.I., Mathematical Methods of Classical Mechanics, Springer Verlag, (1978).

[3] Bertsekas, D. , and Tsitsiklis, J., Neuro-Dynamic Programming, Athena (1997)

[4] Bryson, A.E., and Ho, Y.C., Applied Optimal Control, Taylor and Francis, (1975).

[5] Millennium Prize Problems, Clay Mathematics Institute (2000). 
[6] Drazin, P.G., and W.H. Reid, Hydrodynamic Stability Cambridge University Press, (1981).

[7] Evans, L.C., Partial Differential Equations, American Mathematical Society, (1998).

[8] Hahn, W., Stability of motion, Springer Verlag, (1967).

[9] Hartmann, P. Ordinary differential equations, second ed., Birkhauser, (1982).

[10] Henson, M.A., and D.E Seborg, Eds., Nonlinear Process Control, Prentice-Hall, (1997).

[11] Jawzwinski, A. Stochastic Processes and Filtering Theory, Academic Press, (1970).

[12] Krasovskii, N.N., Problems of the Theory of Stability of Motion, Mir, Moskow, (1959).

[13] Kristiansen, D., Doctoral Thesis, Norwegian University of Science and Technology, Trondheim (2000).

[14] Kristiansen, D., and Egeland, O., Time and spatial discretization methods that preserve passivity properties for systems described by partial differential equations, American Control Conference Chicago, Illinois (2000).

[15] Kristic, M., in Lecture Notes in Control and Information Sciences 244, Springer Verlag (1999).

[16] Lions, P.L., Mathematical Topics in Fluid Mechanics, Clarendon Press (1996).

[17] Lohmiller, W., and Slotine, J.J.E., Metric Analysis for Nonlinear Systems, I.E.E.E. Int. Conf. Control Applications, Dearborn, Michigan, (1996).

[18] Lohmiller, W., and Slotine, J.J.E., On Contraction Analysis for Nonlinear Systems, Automatica, 34(6), (1998).

[19] Lohmiller, W., and Slotine, J.J.E., Stability Analysis and Nonlinear Observer Design for Nonlinear Diffusion Processes, Lecture Notes in Control and Information Sciences 244, Springer Verlag (1999).

[20] Lohmiller, W., and Slotine, J.J.E., European Control Conference, Karlsruhe, Germany (1999).

[21] Lohmiller, W., and Slotine, J.J.E., Nonlinear Process Control Using Contraction Theory, $A$. I. Che. Journal, March 2000.

[22] Lohmiller, W., and Slotine, J.J.E., Global Convergence Rates of Nonlinear Diffusion for Time-Varying images, Scale-Space Theories in Computer Vision, Lecture Notes in Computer Science 1682, Springer Verlag (1999).

[23] Lohmiller, W., and Slotine, J.J.E., Nonlinear PDE Observers for Machine Vision, I.E.E.E. M.M.A.R., Poland (2001) 
[24] Lovelock and Rund, Tensors, Differential Forms, and Variational Principles, Dover Publications (1989).

[25] http://web.mit.edu/ nsl/www/pdeplot.pdf 